\title{
Investigation of the Surface Brightness Model in the Milky Way via Homotopy Perturbation Method
}

\author{
Abdullah A. Alatawi, Mona Aljoufi, Fahad M. Alharbi, Abdelhalim Ebaid \\ Department of Mathematics, Faculty of Sciences, University of Tabuk, Tabuk, KSA \\ Email: halimgamil@yahoo.com
}

How to cite this paper: Alatawi, A.A., Aljoufi, M., Alharbi, F.M. and Ebaid, A. (2020) Investigation of the Surface Brightness Model in the Milky Way via Homotopy Perturbation Method. Journal of Applied Mathematics and Physics, 8, 434-442. https://doi.org/10.4236/jamp.2020.83033

Received: September 17, 2019

Accepted: March 6, 2020

Published: March 9, 2020

Copyright $\odot 2020$ by author(s) and Scientific Research Publishing Inc.

This work is licensed under the Creative

Commons Attribution International

License (CC BY 4.0).

http://creativecommons.org/licenses/by/4.0/

\begin{abstract}
In this paper, a linear delay model in astronomy, called as Ambartsumian equation, is investigated by two different approaches. The first is the approximate homotopy perturbation method (HPM), while the second is a new closed-form solution for this equation. The results are presented through a table and several plots and have been compared with the relevant literature. It is revealed that the present HPM is of higher accuracy than those approximate techniques used in previously published works, when compared with the obtained analytic solution. The convergence of the new analytic solution has been also discussed.
\end{abstract}

\section{Keywords}

Delay, Homotopy Perturbation Method, Analytic Solution

\section{Introduction}

The Ambartsumian equation is used in the theory of surface brightness in the Milky Way [1]. It is a linear delay differential equation given by [2]

$$
z^{\prime}(t)=-z(t)+\frac{1}{q} z\left(\frac{t}{q}\right), \quad q>1,
$$

where $q$ is a constant and

$$
z(0)=\lambda,
$$

where $\lambda$ is also a constant. Existence and uniqueness were discussed in [3]. Although the Adomian decomposition method (ADM) was effective to deal with various types of equations [4]-[18], the HPM55 [19] [20] is preferred here to analyze (1-2). Details of the HPM were introduced by Ayati and Biazar [20]. 
Moreover, it will be shown that the present analysis posses more accuracy over the previous method in the literature.

\section{Application of the HPM}

First, Equation (1) is rewritten as

$$
z^{\prime}(t)=-z(t)+p\left[\frac{1}{q} z\left(\frac{t}{q}\right)\right],
$$

where

$$
z(t)=\sum_{n=0}^{\infty} p^{n} z_{n}(t) .
$$

On substituting (4) into (3), we have

$$
z_{0}^{\prime}(t)+z_{0}(t)+\sum_{n=0}^{\infty} p^{n+1}\left[z_{n+1}^{\prime}(t)+z_{n+1}(t)-\frac{1}{q} z_{n}\left(\frac{t}{q}\right)\right]=0,
$$

which yields

$$
z_{0}^{\prime}(t)+z_{0}(t)=0, \quad z_{0}(0)=\lambda,
$$

and

$$
z_{n+1}^{\prime}(t)+z_{n+1}(t)=\frac{1}{q} z_{n}\left(\frac{t}{q}\right), \quad z_{n+1}(0)=0, \quad n \geq 0 .
$$

Hence,

$$
z_{0}(t)=\lambda \mathrm{e}^{-t}
$$

From (7) and (8), the $1^{\text {st }}$-order system is given as

$$
z_{1}^{\prime}(t)+z_{1}(t)=\frac{\lambda}{q} \mathrm{e}^{-t / q}, \quad z_{1}(0)=0 .
$$

Therefore

$$
z_{1}(t)=\frac{\lambda}{q-1}\left(\mathrm{e}^{-t / q}-\mathrm{e}^{-t}\right)
$$

The $2^{\text {nd }}$-order system is given by

$$
z_{2}^{\prime}(t)+z_{2}(t)=\frac{1}{q} z_{1}\left(\frac{t}{q}\right)=\frac{\lambda}{q(q-1)}\left(\mathrm{e}^{-t / q^{2}}-\mathrm{e}^{-t / q}\right), \quad z_{2}(0)=0 .
$$

By solving the system (11) for $z_{2}(t)$, we have

$$
z_{2}(t)=\frac{\lambda}{\left(q^{2}-1\right)(q+1)}\left[q \mathrm{e}^{-t / q^{2}}-(q+1) \mathrm{e}^{-t / q}+\mathrm{e}^{-t}\right] .
$$

Proceeding as above we obtain the $3^{\text {rd }}$-order system as

$$
z_{3}^{\prime}(t)+z_{3}(t)=\frac{1}{q} z_{2}\left(\frac{t}{q}\right), \quad z_{3}(0)=0,
$$

with the corresponding solution 


$$
Z_{3}(t)=\frac{\lambda}{\left(q^{3}-1\right)(q+1)}\left[q^{3} \mathrm{e}^{-t / q^{3}}-\left(q^{3}+q^{2}+q\right) \mathrm{e}^{-t / q^{2}}+\left(1+q+q^{2}\right) \mathrm{e}^{-t / q}-\mathrm{e}^{-t}\right] .
$$

The calculated higher-order solutions are obtained by MATHEMATICA and then implemented to producing the results in Section 5. From Equation (4), the HPM gives the series solution of Equation (1) as $p \rightarrow 1$ (see Ayati and Biazar [20]) by $z(t)=\sum^{\infty} z_{n}(t)$. This infinite series is approximated by replacing infinity with $n$-term, hence, the approximate solution, denoted by $\psi_{n}(t)$, is given by [19] [20]:

$$
\psi_{n}(t)=\sum_{i=0}^{n-1} z_{i}(t)
$$

The residual $\left|R E_{n}(t)\right|$ is given as

$$
\left|R E_{n}(t)\right|=\left|\psi_{n}^{\prime}(t)+\psi_{n}(t)-\frac{1}{q} \psi_{n}\left(\frac{t}{q}\right)\right|, \quad n \geq 1,
$$

and the approximate solution in Ref. [2] is

$$
\chi_{m}(t)=\lambda\left[1+\sum_{i=1}^{m}\left(\prod_{k=1}^{i}\left(q^{-k}-1\right)\right) \frac{t^{i}}{i !}\right]
$$

\section{Analytic Solution}

Equation (1) can be written as

$$
z^{\prime}(t)=-z(t)+\beta z(\beta t), \quad \beta=\frac{1}{q} .
$$

Assume that

$$
z(t)=\sum_{n=0}^{\infty} c_{n} \mathrm{e}^{-\beta^{n} t}
$$

Accordingly, we have

$$
z^{\prime}(t)=\sum_{n=0}^{\infty}-\beta^{n} c_{n} \mathrm{e}^{-\beta^{n} t},
$$

and

$$
z(\beta t)=\sum_{n=0}^{\infty} c_{n} \mathrm{e}^{-\beta^{n+1} t} .
$$

Inserting Equations (19)-(21) into Equation (18), we get

$$
\sum_{n=0}^{\infty}-\beta^{n} c_{n} \mathrm{e}^{-\beta^{n} t}=-\sum_{n=0}^{\infty} c_{n} \mathrm{e}^{-\beta^{n} t}+\sum_{n=0}^{\infty} \beta c_{n} \mathrm{e}^{-\beta^{n+1} t},
$$

i.e.,

$$
\sum_{n=0}^{\infty}\left[\left(1-\beta^{n+1}\right) c_{n+1}-\beta c_{n}\right] \mathrm{e}^{-\beta^{n+1} t}=0,
$$

and this yields

$$
\left(1-\beta^{n+1}\right) c_{n+1}-\beta c_{n}=0 .
$$


The last equation implies that

$$
c_{n+1}=\left(\frac{\beta}{1-\beta^{n+1}}\right) c_{n}, \quad n \geq 0 .
$$

Accordingly,

$$
\begin{aligned}
& c_{1}=\left(\frac{\beta}{1-\beta}\right) c_{0}, \\
& c_{2}=c_{0}\left(\frac{\beta^{2}}{\prod_{k=1}^{2}\left(1-\beta^{k}\right)}\right), \\
& c_{3}=c_{0}\left(\frac{\beta^{3}}{\prod_{k=1}^{3}\left(1-\beta^{k}\right)}\right), \\
& c_{m}=c_{0}\left(\frac{\beta^{m}}{\prod_{k=1}^{m}\left(1-\beta^{k}\right)}\right), \quad m \geq 1 .
\end{aligned}
$$

Hence

$$
z(t)=c_{0}\left[\mathrm{e}^{-t}+\sum_{n=1}^{\infty}\left(\frac{\beta^{n}}{\prod_{k=1}^{n}\left(1-\beta^{k}\right)}\right) \mathrm{e}^{-\beta^{n} t}\right] .
$$

The initial condition (2), gives $c_{0}$ by

$$
c_{0}=\lambda /\left(1+\sum_{n=1}^{\infty} \frac{\beta^{n}}{\prod_{k=1}^{n}\left(1-\beta^{k}\right)}\right) .
$$

Thus

$$
z(t)=\lambda\left(\frac{\mathrm{e}^{-t}+\sum_{n=1}^{\infty} \frac{\beta^{n} \mathrm{e}^{-\beta^{n} t}}{\prod_{k=1}^{n}\left(1-\beta^{k}\right)}}{1+\sum_{n=1}^{\infty} \frac{\beta^{n}}{\prod_{k=1}^{n}\left(1-\beta^{k}\right)}}\right),
$$

and the convergence of series in (29) is discussed in the next section in detail.

\section{Convergence Analysis}

Definition 1: Let $\left\{f_{n}(x)\right\}$ be a sequence of real functions, each function of which is defined for all $x$ on a real interval $a \leq x \leq b$. For each particular $x$ such that $a \leq x \leq b$ consider the corresponding sequence of real numbers $\left\{f_{n}(x)\right\}$. 
Suppose that the sequence $\left\{f_{n}(x)\right\}$ converges for every $x$ such that $a \leq x \leq b$, and let $f(x)=\lim _{n \rightarrow \infty} f_{n}(x) \quad \forall x \in[a, b]$. Then we say that the sequence of real functions $\left\{f_{n}(x)\right\}$ converges pointwise on the interval $a \leq x \leq b$, and the function $f(x)$ thus defined i called the limit function of the sequence $\left\{f_{n}(x)\right\}$.

Definition 2: Let $\left\{f_{n}(x)\right\}$ be a sequence of real functions, each function of which is defined for all $x$ on a real interval $a \leq x \leq b$. The sequence $\left\{f_{n}(x)\right\}$ is said to converge uniformly to $f(x)$ on $a \leq x \leq b$ if, given any $\varepsilon>0$, there exists $N>0$ (which depends only upon $\varepsilon$ ) such that $\left|f_{n}(x)-f(x)\right|<\varepsilon$ $\forall n>N, \forall x \in[a, b]$.

Theorem 1: Let $\left\{f_{n}(x)\right\}$ be a sequence of real functions converges uniformly to $f(x)$ on $a \leq x \leq b$ and suppose that each function $f_{n}(x)(n=1,2,3, \cdots)$ is continuous on $a \leq x \leq b$, then the limit function $f(x)$ is continuous on $a \leq x \leq b$.

\section{Theorem 2: Weierstrass M-Test}

1) Let $\left\{M_{n}\right\}$ be a sequence of positive constants such that the series of constants $\sum_{n=1}^{\infty} M_{n}$ converge.

2) Let $\sum_{n=1}^{\infty} u_{n}(x)$ be a series of real functions such that $\left|u_{n}(x)\right| \leq M_{n}$ $\forall x \in[a, b]$ for each $n=1,2,3, \cdots$.

Then the series $\sum_{n=1}^{\infty} u_{n}(x)$ converges uniformly on $x \in[a, b]$.

Theorem 3: From (29), Let $M_{n}=\frac{\beta^{n}}{\prod_{k=1}^{n}\left(1-\beta^{k}\right)}$, then $\left\{M_{n}\right\}$ is a sequence of positive constants and the series $\sum_{n=1}^{\infty} M_{n}$ converges.

Proof: Since $\beta=1 / q$ and $q>1$, then $\beta$ is a positive constant and $0<\beta<1$. Also, the expression $\left(1-\beta^{k}\right)$ is always positive, where $0<\beta^{k}<1$. Therefore, $\left\{M_{n}\right\}$ is a sequence of positive constants. To prove convergence of the series $\sum_{n=1}^{\infty} M_{n}$, we have from the ratio test that

$$
\begin{aligned}
\lim _{n \rightarrow \infty}\left|\frac{M_{n+1}}{M_{n}}\right| & =\lim _{n \rightarrow \infty}\left|\frac{\beta^{n+1}}{\prod_{k=1}^{n+1}\left(1-\beta^{k}\right)} \times \frac{\prod_{k=1}^{n}\left(1-\beta^{k}\right)}{\beta^{n}}\right|=\lim _{n \rightarrow \infty}\left|\frac{\beta}{1-\beta^{n+1}}\right|, \\
& =\beta \times \lim _{n \rightarrow \infty}\left|\frac{1}{1-\beta^{n+1}}\right|=\beta \times 1<1
\end{aligned}
$$

which proves the convergence of the series $\sum_{n=1}^{\infty} M_{n}$.

Theorem 4: The solution $z(t)$ given by (29) converges uniformly on the whole domain $0 \leq t<\infty$.

Proof: From the previous theorem, it we showed that $\left\{M_{n}\right\}$ is a sequence of positive constants and the series $\sum_{n=1}^{\infty} M_{n}$ converges. This meets the first requirement of the Weierstrass M-Test in theorem 2 above. Since $\sum_{n=1}^{\infty} M_{n}$ converges, we suppose that its sum equals $M^{*}$, i.e., $\sum_{n=1}^{\infty} M_{n}=M^{*}$. In order to satisfy the second requirement of theorem 2, we rewrite the solution (29) in terms of a new variable $x$, where $x=\mathrm{e}^{-t}$, as: 


$$
z(x)=\frac{\lambda}{1+M^{*}}\left(x+\sum_{n=1}^{\infty} M_{n} x^{\beta^{n}}\right), \quad 0<x \leq 1 .
$$

To prove convergence of $z(x)$ in (31), it is sufficient to prove the convergence of the series $\sum_{n=1}^{\infty} u_{n}=\sum_{n=1}^{\infty} M_{n} x^{\beta^{n}}$. At this stage, we have from $u_{n}=M_{n} x^{\beta^{n}}$ that

$$
\left|u_{n}\right|=\left|M_{n} x^{\beta^{n}}\right|=\left|M_{n}\right|\left|x^{\beta^{n}}\right| \leq M_{n},
$$

where $\left|x^{\beta^{n}}\right| \leq 1 \quad \forall n=1,2,3, \cdots$, and this completes the proof.

\section{Validation of Numerical Results}

The HPM and another direct approach have been applied in the previous sections to obtain the approximate solutions and the analytic solution, respectively, in terms of exponential functions with negative powers. The convergence of the analytic solutions was discussed in Section 4. The obtained approximate solutions by the HPM are to be analyzed here in view of the analytic solution (29) to stand on their accuracy. This can be achieved via performing comparisons with the results in the literature. The comparisons between the present results and those of Ref. [2] and [21] are presented in Table 1. The present HPM is of higher accuracy as observed from Table 1 . This indicates the advantages of the current approach over those in the literature when analyzing the Ambartsumian equation.

The behavior of $\left|R E_{11}\right|$ is displayed in Figure $1(1.1 \leq q \leq 1.4)$, Figure 2 $(1.4 \leq q \leq 2.0)$, Figure $3(2.0 \leq q \leq 3.0)$, and Figure $4(3.0 \leq q \leq 10)$ for $t \in[0,100]$. It can be seen from these figures that the maximum values of $\left|R E_{11}\right|$ are $6 \times 10^{-2}, 6 \times 10^{-3}, 2 \times 10^{-9}$, and $1.5 \times 10^{-16}$, respectively. This proves the efficiency of the HPM over the previous ones in [2] and [21].

\section{Conclusion}

The HPM was applied to solve the Ambartsumian equation in terms of exponential functions. The obtained solution was valid in the whole domain, while the corresponding solution in the literature [2] was only valid in sub-domains.

\begin{tabular}{|c|c|c|c|c|c|c|}
\hline \multirow[b]{2}{*}{$t$} & \multirow[b]{2}{*}{ Ref. [2] } & \multirow[b]{2}{*}{ HATM [21] } & \multicolumn{4}{|c|}{ Present } \\
\hline & & & $\begin{array}{c}\text { HPM } \\
\text { (4-term) }\end{array}$ & $\begin{array}{c}\text { HPM } \\
\text { (7-term) }\end{array}$ & $\begin{array}{c}\text { HPM } \\
\text { 9-term) }\end{array}$ & $\begin{array}{l}\text { Analytic solution } \\
\text { (Equation (29)) }\end{array}$ \\
\hline 0.0 & 1 & 1 & 1 & 1 & 1 & 1 \\
\hline 0.5 & 0.8727825992 & 0.8727825992 & 0.8728718032 & 0.8729409264 & 0.8729409265 & 0.8729409265 \\
\hline 1.0 & 0.7694328044 & 0.7694328044 & 0.7709321110 & 0.7717847777 & 0.7717847885 & 0.7717847885 \\
\hline 1.5 & 0.6788327993 & 0.6788327993 & 0.6865930139 & 0.6899347736 & 0.6899349261 & 0.6899349261 \\
\hline 2.0 & 0.5898647673 & 0.5898647673 & 0.61449731667 & 0.6227074556 & 0.6227083998 & 0.6227083998 \\
\hline
\end{tabular}

Table 1. Comparison of the present results with the corresponding results in the literature. 


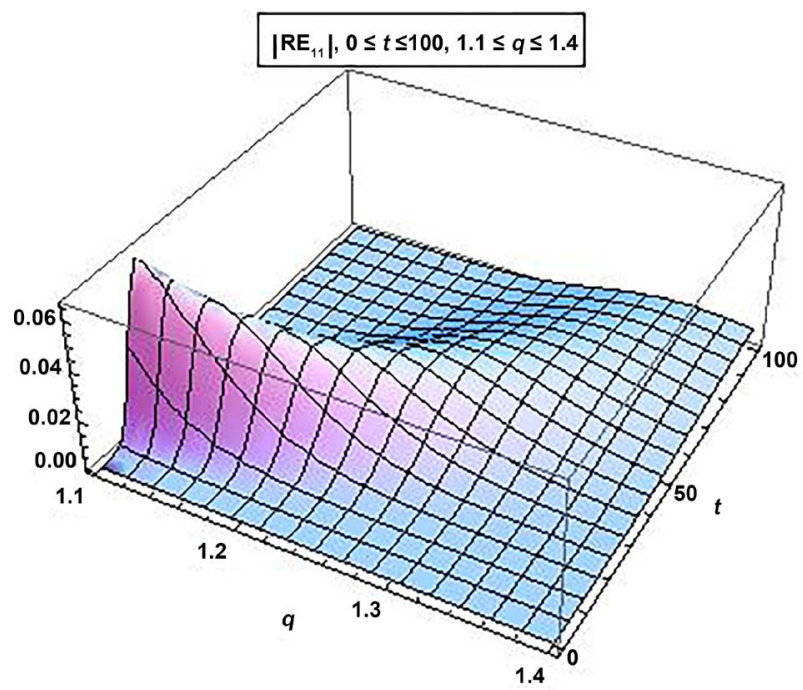

Figure 1. Residual for $1.1 \leq q \leq 1.4$.

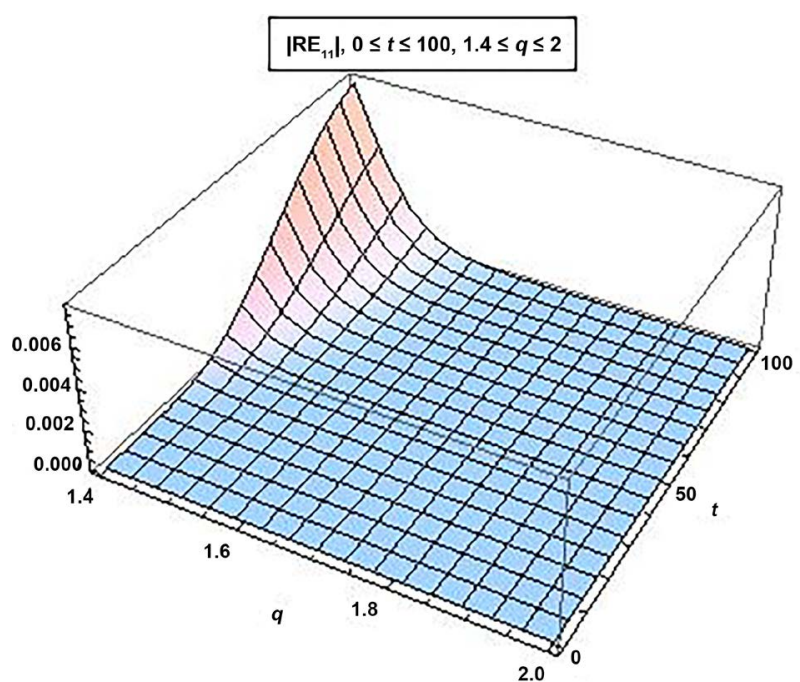

Figure 2. Residual for $1.4 \leq q \leq 2$.

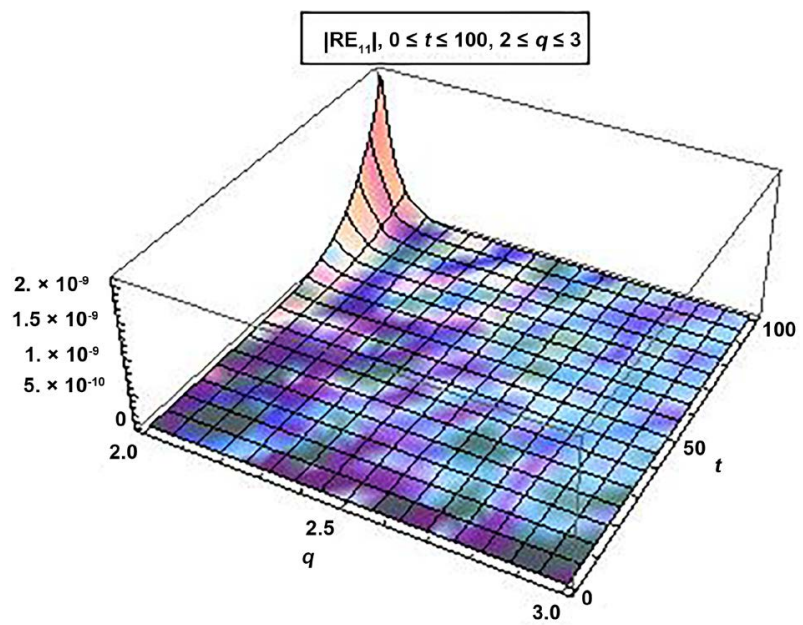

Figure 3. Residual for $2 \leq q \leq 3$. 


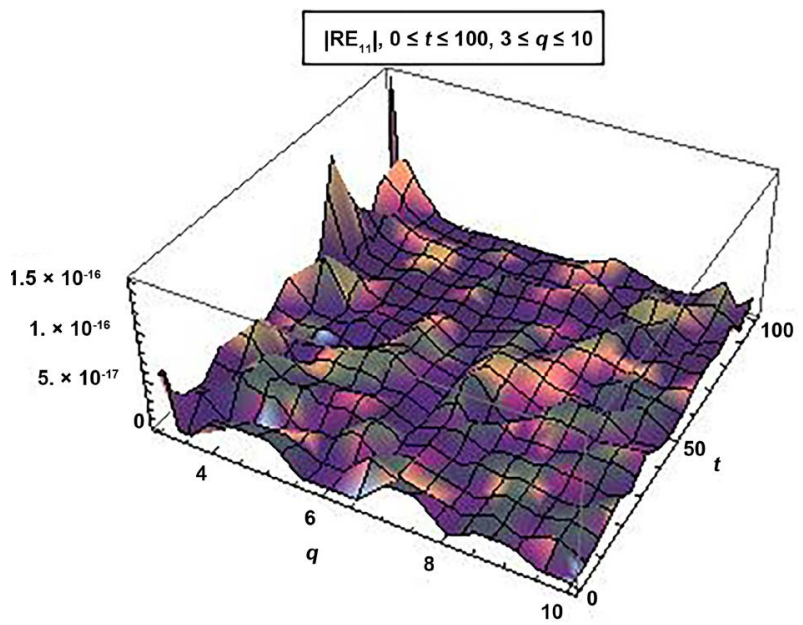

Figure 4. Residual for $3 \leq q \leq 10$.

Moreover the obtained residual tends to zero as the $q$ increases. In view of references [2] and [21], the present HPM is of higher accuracy when compared with the obtained exact solution.

\section{Conflicts of Interest}

The authors declare no conflicts of interest regarding the publication of this paper.

\section{References}

[1] Ambartsumian, V.A. (1994) On the Fluctuation of the Brightness of the Milky Way. Doklady Akademii Nauk SSSR, 44, 223-226.

[2] Patade, J. and Bhalekar, S. (2017) On Analytical Solution of Ambartsumian Equation. National Academy Science Letters, 40, 291-293. https://doi.org/10.1007/s40009-017-0565-2

[3] Kato, T. and McLeod, J.B. (1971) The Functional-Differential Equation $y^{\prime}(x)=a y(\lambda x)+b y(x)$. Bulletin of the American Mathematical Society, 77, 891-935. https://doi.org/10.1090/S0002-9904-1971-12805-7

[4] Adomian, G. and Rach, R. (1985) On the Solution of Algebraic Equations by the Decomposition Method. Journal of Mathematical Analysis and Applications, 105, 141-166. https://doi.org/10.1016/0022-247X(85)90102-7

[5] Adomian, G. and Rach, R. (1985) Algebraic Equations with Exponential Terms. Journal of Mathematical Analysis and Applications, 112, 136-140. https://doi.org/10.1016/0022-247X(85)90280-X

[6] Adomian, G. and Rach, R. (1986) Algebraic Computation and the Decomposition Method. Kybernetes, 15, 33-37. https://doi.org/10.1108/eb005727

[7] Wazwaz, A.M. (2005) Adomian Decomposition Method for a Reliable Treatment of the Bratu-Type Equations. Applied Mathematics and Computation, 166, 652-663. https://doi.org/10.1016/j.amc.2004.06.059

[8] Aly, E.H., Ebaid, A. and Rach, R. (2012) Advances in the Adomian Decomposition Method for Solving Two-Point Nonlinear Boundary Value Problems with Neumann Boundary Conditions. Computers \& Mathematics with Applications, 63, 
1056-1065. https://doi.org/10.1016/j.camwa.2011.12.010

[9] Chun, C., Ebaid, A., Lee, M. and Aly, E. (2012) An Approach for Solving Singular Two Point Boundary Value Problems: Analytical and Numerical Treatment. ANZIAM Journal, 53, 21-43. https://doi.org/10.21914/anziamj.v53i0.4582

[10] Yang, X.-J., Baleanu, D. and Zhong, W.-P. (2013) Approximate Solutions for Diffusion Equations on Cantor Space-Time. Proceedings of the Romanian Academy-Series $A, 14,127-133$.

[11] Lin, W.Y. and Chen, C.-K. (2014) Modified Adomian Decomposition Method for Double Singular Boundary Value Problems. Romanian Journal of Physics, 59, 443-453.

[12] Triki, H. (2014) Solitons and Periodic Solutions to the Dissipation-Modified KdV Equation with Time-Dependent Coefficients. Romanian Journal of Physics, 59, 421-432.

[13] Jafari, H., Haghbin, A., Hesam, S. and Baleanu, D. (2014) Solving Partial q-Differential Equations within Reduced q-Differential Transformation Method. Romanian Journal of Physics, 59, 399-407.

[14] Fatoorehchi, H. and Abolghasemi, H. (2014) Finding All Real Roots of a Polynomial by Matrix Algebra and the Adomian Decomposition Method. Journal of the Egyptian Mathematical Society, 22, 524-528. https://doi.org/10.1016/j.joems.2013.12.018

[15] Cristescu, I.A. (2015) Decomposition Method for Neutron Transport Equation. Romanian Journal of Physics, 60, 179-189.

[16] Alshaery, A. and Ebaid, A. (2017) Accurate Analytical Periodic Solution of the Elliptical Kepler Equation Using the Adomian Decomposition Method. Acta Astronautica, 140, 27-33. https://doi.org/10.1016/j.actaastro.2017.07.034

[17] Gaber, A.A. and Ebaid, A. (2018) Analytical Study on the Slip Flow and Heat Transfer of Nanofluids over a Stretching Sheet Using Adomian's Method. Romanian Reports in Physics, 70, 110.

[18] Bakodah, H.O. and Ebaid, A. (2018) The Adomian Decomposition Method for the Slip Flow and Heat Transfer of Nanofluids over a Stretching/Shrinking Sheet. Romanian Reports in Physics, 70, 115.

[19] Patra, A. and Saha Ray, S. (2014) Homotopy Perturbation Sumudu Transform Method for Solving Convective Radial Fins with Temperature-Dependent Thermal Conductivity of Fractional Order Energy Balance Equation. International Journal of Heat and Mass Transfer, 76, 162-170. https://doi.org/10.1016/j.ijheatmasstransfer.2014.04.020

[20] Ayati, Z. and Biazar, J. (2015) On the Convergence of Homotopy Perturbation Method. Journal of the Egyptian Mathematical Society, 23, 424-428. https://doi.org/10.1016/j.joems.2014.06.015

[21] Kumar, D., Singh, J., Baleanu, D. and Rathore, S. (2018) Analysis of a Fractional Model of the Ambartsumian Equation. The European Physical Journal Plus, 133, 259. https://doi.org/10.1140/epjp/i2018-12081-3 